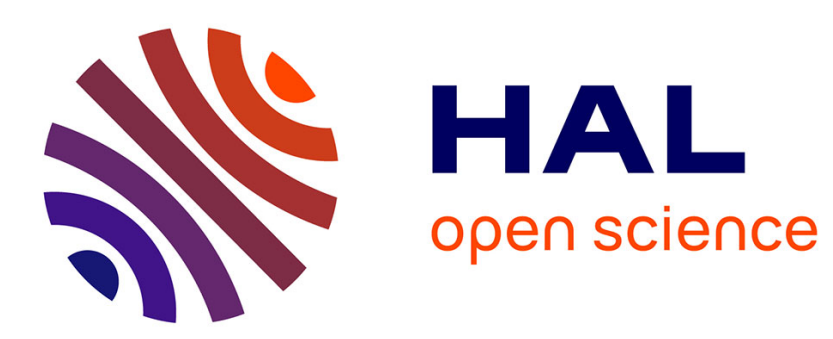

\title{
An evaluation framework and a benchmark for multi/hyperspectral image compression
}

Jonathan Delcourt, Alamin Mansouri, Tadeusz Sliwa, Yvon Voisin

\section{To cite this version:}

Jonathan Delcourt, Alamin Mansouri, Tadeusz Sliwa, Yvon Voisin. An evaluation framework and a benchmark for multi/hyperspectral image compression. Computer Vision Graphics and Image Processing, 2011, 1 (1), pp.55-71. 10.4018/ijcvip.2011010105 . hal-00637853

\section{HAL Id: hal-00637853 \\ https://u-bourgogne.hal.science/hal-00637853}

Submitted on 3 Nov 2011

HAL is a multi-disciplinary open access archive for the deposit and dissemination of scientific research documents, whether they are published or not. The documents may come from teaching and research institutions in France or abroad, or from public or private research centers.
L'archive ouverte pluridisciplinaire HAL, est destinée au dépôt et à la diffusion de documents scientifiques de niveau recherche, publiés ou non, émanant des établissements d'enseignement et de recherche français ou étrangers, des laboratoires publics ou privés. 


\title{
An Evaluation Framework and a Benchmark For Multi/Hyperspectral Image Compression
}

\author{
Jonathan Delcourt, Alamin Mansouri, Tadeusz Sliwa and Yvon Voisin \\ University of Burgundy \\ Laboratoire Le2i, BP 16 Route des Plaines de l'Yonne, \\ 89010 Auxerre Cedex, France \\ Email: jonathan.delcourt@u-bourgogne.fr
}

\begin{abstract}
In this paper, we investigate different approaches for multi/hyperspectral image compression. In particular, we compare the classic Multi-2D compression approach and two different implementations of a 3D approach (Full 3D and Hybrid) with regards to variations in spatial and spectral dimensions. All approaches are combined with a spectral Principal Component Analysis (PCA) decorrelation stage to optimize performance. For consistent evaluation, we propose a larger comparison framework than the conventionally used PSNR, including eight metrics divided into three families. We also discuss the time and memory consumption difference between the three compression approaches. The results show the weaknesses and strengths of each approach.
\end{abstract}

\section{Introduction}

A hyper/multispectral imaging system splits the light spectrum into more than three frequency bands (dozens to hundreds) and records each of the images separately as a set of monochrome images. This type of technique increases the number of acquisition channels in the visible spectrum and extends channel acquisition to the light that is outside the sensitivity of the human eye. Such systems offer several advantages over conventional RGB imaging and have, therefore, attracted increasing interest in the past few years. However, multispectral uncompressed images, in which a single image-band may occupy hundreds of megabytes, often require high capacity storage. Compression is thus necessary to facilitate both the storage and the transmission of multispectral images.

Generally, a multispectral image is represented as a 3D cube with one spectral and two spatial dimensions. The fact that a multispectral image consists of a series of narrow and contiguously spectral bands of the same scene produces a highly correlated sequence of images. This particularity differentiates multispectral images from volumetric ones with three isotropic spatial dimensions, and also from videos with one temporal and two spatial dimensions. Conventional compression methods are not optimal for multispectral image compression, which is why compression algorithms need to be adapted to this type of image.

One of the most efficient compression methods for monochrome images compression is the JPEG $2000^{1}$ (Boliek, Christopoulos, \& Majani, 2000 ; Boliek, Majani, Houchin, Kasner, \& Carlander, 2000 ; Christopoulos, Skodras, \& Ebrahimi, 2000 ; Taubman, 2000 ; Taubman, Marcellin, \& Rabbani, 2002). Its extension to multi/hyperspectral images yields to different approaches. These approaches depend on the manner of which one consider the multi/hyperspectral cube after the decorrelation stage (figure 1):

- in the Multi-2D approach, each image band of the multi/hyperspectral image is considered separately (2D wavelets + 2D SPIHT) (Du \& Fowler, 2007 ; Kaarna, Toivanen, \& Keranen, 2006 ; Mielikainen \& Kaarna, 2002),

- the whole cube is considered as input leading to two main implementations: the Hybrid approach (3D wavelets + 2D SPIHT), as used in (Penna, Tillo, Magli, \& Olmo, 2006), and the Full 3D approach (3D wavelets + 3D SPIHT). For this latter we used an anisotropic 3D wavelets decomposition. Many works in literature have explored the 3D wavelet transform for multi/hyperspectral image compression but they only use isotropic 3D wavelets (same type of wavelets following all directions) (Kaarna, Zemcik, Kaelviainen, \& Parkkinen, 1998 ; Kaarna \& Parkkinen, 2000b, 2000a ; Kaarna, 2001 ; Kaarna et al., 2006 ; Kim, Xiong, \& Pearlman, 2000 ; Lim, Sohn, \& Lee, 2001 ; Mielikainen \& Kaarna, 2002 ; Penna et al., 2006 ; Tang, Cho, \& Pearlman, 2003), but only Kaarna and Parkkinen describe a compression method based on an anisotropic wavelets decomposition in (Kaarna \& Parkkinen, 1998).

We tested the three compression approaches with the same lifting scheme wavelet transform and compared them. To provide a more objective benchmark, we propose a framework of evaluation composed of seven metrics in addition to the classic PSNR. These metrics evaluate the quality of reconstruction in terms of signal, spectral reflectance and perceptive aspects.

In the next section, we provide a short overview of how we use the principal composant analysis algorithm (PCA) (Pearson, 1901) within the three compression approaches, before describing them into the second section. The third section introduces the framework of comparison by splitting

\footnotetext{
${ }^{1}$ http://www.jpeg.org
} 


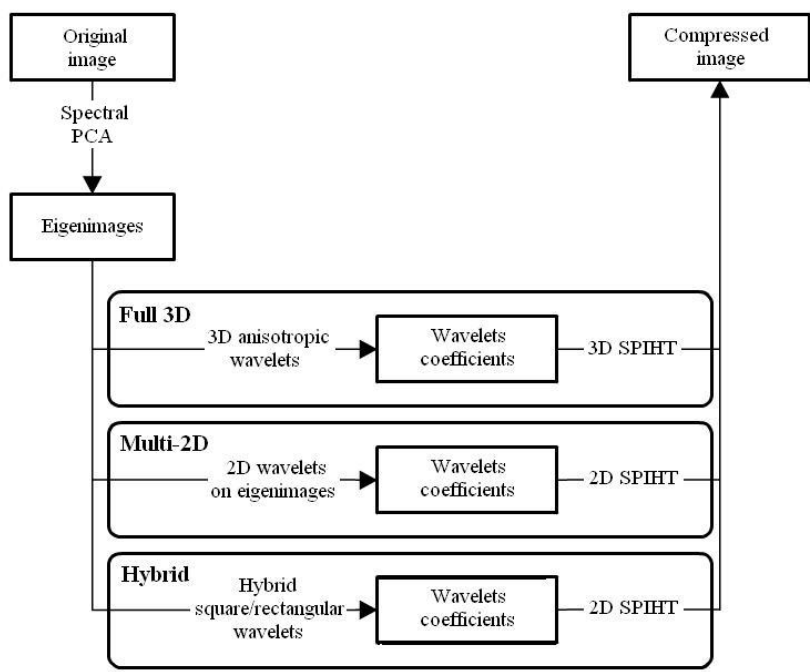

Figure 1. Graphical representation of the three compression approaches.

the metrics into three families and gives the explicit formula for each metric. We discuss our experiments and their results in the fourth section and highlight the strengths and weaknesses of the three approaches. In order to take the algorithmic aspects of these strategies into account, we will also discuss them in terms of time and memory consumption in the fourth section. Conclusions are presented in the last section.

\section{Compression approaches}

For the implementation of the three approaches we chose to use the wavelets of JPEG 2000 standard because it is a reference for 2D compression. The JPEG 2000 standard wavelets are "Le Gall 5/3" for lossless compression and "Cohen-Daubechies-Feauveau 9/7" (or CDF 9/7) for lossy compression. In our case we perform lossy compression, so we will use the CDF 9/7 wavelet.

As previously reported, multispectral images have a high correlation between image-bands. To achieve the best compression ratios it is necessary to take this correlation into account.

\section{PCA decorrelation}

In order to optimize multi/hyperspectral image compression, a decorrelation step is often used. In this context, several methods have been developed. Classic algorithms are based on vector quantization (Gupta \& Gersho, 1992), wavelets or Hybrid methods, such as DPCM-DCT (Abousleman, Marcellin, \& Hunt, 1995), KLT-DCT (Saghri \& Tescher, 1991) and PCA (KLT). The PCA has been shown to be one of the most efficient spectral decorrelators (Ready \& Wintz, 1973) and is used in many compression methods.

Epstein et al. propose in (Epstein, Hingorani, Shapiro, \& Czigler, 1992) a method for landsat thematic mapper multi- spectral imagery. The method first removes inter-band correlation by PCA to produce principle components of seven landsat bands. The principle components are then compressed using wavelet and lossless compression techniques like run length encoding. Harsanyi and Chang (Harsanyi \& Chang, 1994) applied PCA to hyperspectral images to reduce data dimensionality, suppress undesired or interfering spectral signatures, and classify the spectral signatures of interest. In (Mielikainen \& Kaarna, 2002), Mielikäinen and Kaarna applied PCA to reduce correlation among spectral bands, but they only selected a small number of spectra from the image for the calculation of the eigenvectors. They then applied integer wavelet transform to the residual image to concentrate energy and reduce entropy. Du and Fowler implemented PCA along with JPEG 2000 for hyperspectral image compression (Du \& Fowler, 2007). They assumed that PCA would help in spectral decorrelation and JPEG 2000 would help in compression. They found that the method performed better than the combination scheme of wavelet for spectral decorrelation and JPEG 2000 for compression. They tested both methods and found that, for rate-distortion and information preservation, PCA with JPEG 2000 outperformed JPEG 2000 alone.

Other spectral decorrelators based on PCA may be used. In (Chang, Cheng, \& Chen, 2000), adaptive KLT is used for decorrelation. The original image is divided into proper regions, and transforms each image data-set region by the corresponding transformation function. The results of their simulations show that the performance of adaptive KLT is better than KLT alone. In (Gu, Zhang, \& Zhang, 2002) Gu et al. proposed a Kernel Based Nonlinear Subspace Projection (KNSP) method followed by kernel PCA. They partitioned the full data space into different subspaces. Next, they used Kernel PCA for feature selection based on class separatibility criteria. The authors claim that the method is more suitable for feature extraction than linear PCA and segmented linear component transformation, particularly when hyperspectral data have non-linear characteristics.

Some compression algorithms are optimised for specific applications (classification, visualisation, dimension reduction, etc.). For this they will use PCA variants. In our case we simply seek to compress, without knowledge of the final image utilisation, which is why we use classical PCA, which does not favor any particular use.

In our experiments, we applied PCA to the original multi/hyperspectral image in the spectral dimension. As a result, we obtain a new multiband image in the transform domain in which the spectral correlation is reduced. The image-bands in the transform domain were sorted with decreasing variance (according to the values of the eigenvalues). We finally applied the three compression approaches to all bands of the transformed image, unlike in dimension reduction (Harsanyi \& Chang, 1994 ; Mielikainen \& Kaarna, 2002 ; Du \& Fowler, 2007 ; Gu et al., 2002) where only a few bands were selected. This procedure allowed us to preserve the maximum amount of image information. 


\section{First approach - Multi-2D}

This approach consists of applying the same $2 \mathrm{D}$ wavelets transform on each band of the resulting PCA image. Because of PCA, the resulting image has decreasing energy bands. In order to take this fact into account, it is preferable to weigh each band. For weights, we define the energy $E$ of each band as in the formula:

$$
E=\frac{\sqrt{\sum_{x, y} I_{\lambda}(x, y)^{2}}}{X Y}
$$

where $I_{\lambda}$ is the image band at the $\lambda$ wavelength, $X$ and $Y$ are its dimensions, and $x$ and $y$ are the position of a pixel in the band.

Afterwards, we apply a 2D SPIHT (Said \& Pearlman, 1996) coding to each band of the wavelet transform results to achieve compression.

\section{Second approach - Full 3D}

The Full 3D approach consists of considering the whole multi/hyperspectral image cube as an input for a 3D wavelet transform. In our case the input is the result of the PCA. Then a 3D extension of SPIHT encoder is applied. The 3D SPIHT encoder of Kim et al. (Kim et al., 2000) is appropriate to the 3D block shape of the decomposition. Dragotti et al. (Dragotti, Poggi, \& Ragozini, 2000) also propose a 3D extension of SPIHT for multispectral image compression, but this encoder works like a temporal compensator and is more appropriate for video coding.

However, since the spectral dimension of the multispectral images is lower than the two other spatial dimensions and since following this dimension the correlation is higher, it is appropriate to use a different type of wavelets for this dimension. In (Kaarna \& Parkkinen, 1998) Kaarna and Parkkinen recommend a short wavelets basis as a good choice for spectral wavelets. This recommendation is confirmed by the results obtained by Mansouri et al. in (Mansouri, Sliwa, Hardeberg, \& Voisin, 2008) in which the authors propose the Haar lifting scheme wavelets basis as an appropriate short support basis for reflectance representation and estimation from multispectral images.

Technically speaking, it is possible to use two methods to apply the Full 3D wavelets transform. The classic square wavelets transform method produces a multidimensional wavelets transform by applying one level of the onedimensional (1D) transform separately in all dimensions and then iterating this procedure on the approximation cube. The other way of obtaining a multidimensional wavelets transform consists first of computing all the desired decomposition iterations along one dimension, then all the desired iterations on the next dimension, and so on. This method is called the hybrid rectangular/square wavelets transform. We depict these two principles in Fig.2. We use the classic square wavelets transform in the Full 3D approach followed by 3DSPIHT algorithm and the hybrid rectangular/square wavelets transform is used in the Hybrid approach.

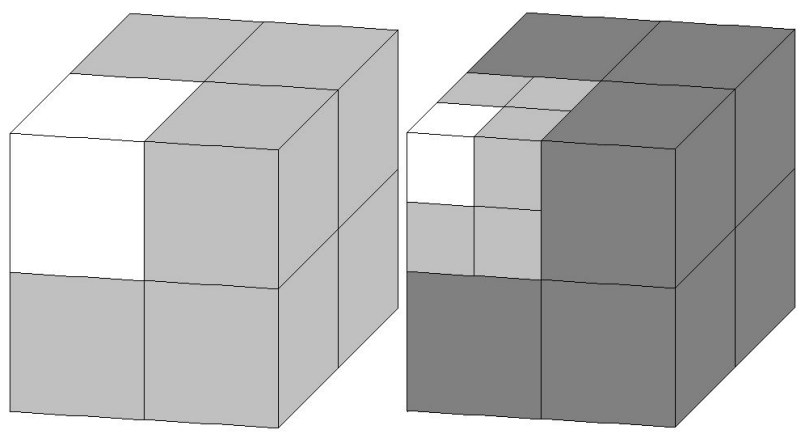

(a)

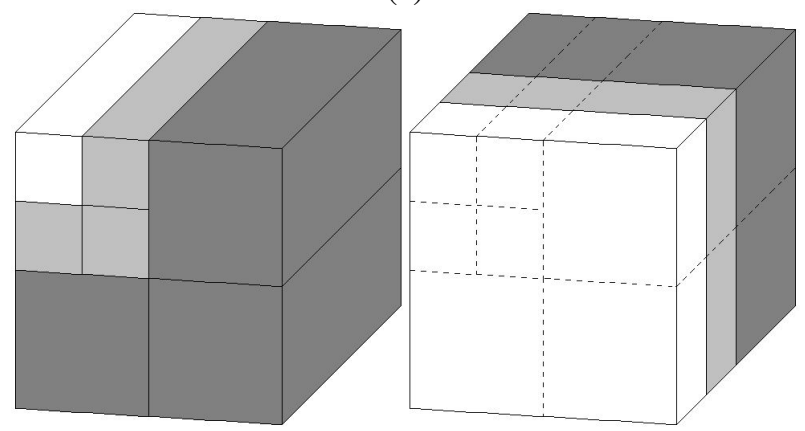

(b)

Figure 2. Graphical representation of the two ways of 3D wavelets decomposition: (a) square decomposition by its first and second steps; (b) hybrid rectangular/square wavelets decomposition with two spatial decompositions followed by two spectral decomposition.

This Full 3D implementation of the wavelets transform takes into account the high spectral correlation of the image and its anisotropy.

\section{Third approach - Hybrid}

The third approach consists of applying a Full 3D wavelets transform on the PCA result as in the Full 3D approach. But the square wavelets transform is replaced by a hybrid rectangular/square wavelets (Fig.2) as used by Penna et al. in (Penna et al., 2006). This wavelets transform takes into account the multispectral image properties. But the fact that this wavelets transform has two differentiated stages (spatial transform is followed by spectral transform) allows its result to be considered as multiple $2 \mathrm{D}$ plans. For this reason we apply 2D SPIHT coding on each resulting band to achieve compression as in the Multi-2D approach. In order to take the difference of energy bands into account we weigh each band with its energy $E$ as in equation (1).

\section{Compression evaluation framework}

When lossy compression methods are used, quality measurements are necessary to evaluate performance. According to Eskicioglu (Eskicioglu \& Fisher, 1995), the main problem in evaluating lossy compression techniques is the difficulty 
of describing the nature and importance of the degradation on the reconstructed image. Furthermore, in the case of ordinary $2 \mathrm{D}$ images, a metric often has to reflect the visual perception of a human observer. This is not the case for hyperspectral images, which are first used through classification or detection algorithms. Therefore, metrics must correspond to applications. This is why instead of evaluating compression performances according to one metric or one type of metric, we propose the utilization of eight known metrics belonging to three categories to do so. We call this a framework for compression evaluation. In (Christophe, Léger, \& Mailhes, 2005), Christophe et al. show that the use of a set of metrics is more relevant than using just one.

The metrics we propose can be divided into three families: signal processing isotropic extended metrics (PSNR, RRMSE, MAE and MAD), spectral oriented metrics $\left(F_{\lambda}\right.$, MSA and GFC), and an advanced statistical metric taking some perceptive aspects into account (UIQI). We use the PSNR in order to facilitate comparison with other methods, since it is the metric most employed in image compression.

In the following sections this notation will be used: $I$ is the original multispectral image and $\tilde{I}$ is the reconstructed multispectral image. The multispectral images are represented in three-dimensional matrix form: $I(x, y, \lambda), x$ is the pixel position in a row, $y$ the number of the row and $\lambda$ the spectral band. $n_{x}, n_{y}, n_{\lambda}$ respectively the number of pixels in a row, the number of rows and the number of spectral bands.

We also introduce the notation $I(x, y, \cdot)$ stands for $I(x, y, \cdot)=\left\{I(x, y, \lambda) \mid 1 \leq \lambda \leq n_{\lambda}\right\}$. In this case $I(x, y, \cdot)$ corresponds to a vector of $n_{\lambda}$ components.

For simplification, we note $I(x, y, \lambda)$ and $\tilde{I}(x, y, \lambda)$ by $I$ and $\tilde{I}$, and also $\sum_{x=1}^{n_{x}} \sum_{y=1}^{n_{y}} \sum_{\lambda=1}^{n_{\lambda}} I$ by $\sum_{x, y, \lambda} I$.

\section{Signal processing isotropic extended metrics}

These metrics come from classic statistical measures. They do not take into account the difference between spatial and spectral dimensions. The structural aspect of errors does not appear.

Relative root mean square error (RRMSE). It is a classic statistical measure based on $\operatorname{MSE}\left(\mathcal{L}_{p}\right.$ norm $)$ with a normalization by the signal level.

$$
\operatorname{RRMSE}(I, \tilde{I})=\sqrt{\frac{1}{n_{x} n_{y} n_{\lambda}} \sum_{x, y, \lambda}\left(\frac{I-\tilde{I}}{I}\right)^{2}}
$$

Mean absolute error (MAE).

$$
\operatorname{MAE}(I, \tilde{I})=\frac{1}{n_{x} n_{y} n_{\lambda}} \sum_{x, y, \lambda}|I-\tilde{I}|
$$

Maximum absolute distortion (MAD). The MAD is used to give a upper bound on the entire image.

$$
\operatorname{MAD}(I, \tilde{I})=\max \{|I-\tilde{I}|\}
$$

\section{Spectral oriented metrics}

These metrics are specially adapted for the multispectral field.

Goodness of fit coefficient (GFC).

$$
\operatorname{GFC}(I, \tilde{I})=\frac{\left|\sum_{j} R_{I}\left(\lambda_{j}\right) R_{\tilde{I}}\left(\lambda_{j}\right)\right|}{\left|\sum_{j}\left[R_{I}\left(\lambda_{j}\right)\right]^{2}\right|^{\frac{1}{2}}\left|\sum_{j}\left[R_{\tilde{I}}\left(\lambda_{j}\right)\right]^{2}\right|^{\frac{1}{2}}}
$$

where $R_{I}\left(\lambda_{j}\right)$ is the original spectrum at wavelength $\lambda_{j}$ and $R_{\tilde{I}}\left(\lambda_{j}\right)$ is the reconstructed spectrum at the wavelength $\lambda_{j}$.

The GFC is bounded, facilitating its understanding. We have $0 \leq \mathbf{G F C} \leq 1$. The reconstruction is very good for GFC $>0.999$ and perfect for a GFC $>0.9999$.

Spectral fidelity $\mathbf{F}_{\lambda}$. This metric was developed by Eskicioglu (Eskicioglu \& Fisher, 1993). We define fidelity by :

$$
\mathbf{F}(I, \tilde{I})=1-\frac{\sum_{x, y, \lambda}[I-\tilde{I}]^{2}}{\sum_{x, y, \lambda}[I]^{2}}
$$

We will take into account the following adaptation focus on spectral dimension to obtain spectral fidelity:

$$
\mathbf{F}_{\lambda}(I, \tilde{I})=\min _{x, y}\{F(I(x, y, \cdot), \tilde{I}(x, y, \cdot))\}
$$

Maximum spectral angle (MSA). The MSA is a metric used in (Keshava \& Mustard, 2002). The spectral angle represents the angle between two spectra viewed as vectors in an $n_{\lambda}$-dimensional space.

$$
\mathbf{S A}_{x, y}=\cos ^{-1}\left(\frac{\sum_{\lambda} I . \tilde{I}}{\sqrt{\sum_{\lambda} I^{2} \sum_{\lambda} \tilde{I}^{2}}}\right)
$$

In our case we take the maximum of SA with:

$$
\mathbf{M S A}=\max _{x, y}\left(\mathbf{S} \mathbf{A}_{x, y}\right)
$$

\section{Universal image quality index (UIQI)}

The UIQI was developed by Wang (Wang \& Bovik, 2002) for monochrome images. This metric uses structural distortion rather than error sensibility. It is an advanced statistical metric. The UIQI is based on three factors: loss of correlation, luminance distortion and contrast distortion.

$$
Q(U, V)=\frac{4 \sigma_{U V} \mu_{U} \mu_{V}}{\left(\sigma_{U}^{2}+\sigma_{V}^{2}\right)\left(\mu_{U}^{2}+\mu_{V}^{2}\right)}
$$


with $\sigma_{U V}$ the cross correlation $\mathbb{E}\left[\left(U-\mu_{U}\right)\left(V-\mu_{V}\right)\right], \mu$ is the mean and $\sigma^{2}$ the variance. The result is bounded by: $-1 \leq Q \leq 1$.

The UIQI can be applied in three different ways, on each band, on each spectrum of the image or on both. We use it on each spectral band of the image as follows:

$$
Q_{x, y}=\min _{\lambda}\{Q(I(\cdot, \cdot, \lambda), \tilde{I}(\cdot, \cdot, \lambda))\}
$$

\section{Experiments and results}

We conducted our experiments on the largely used AVIRIS $^{2}$ images SanDiego, JasperRidge and MoffettField (Fig. 3). These images represent very different landscapes, JasperRidge represent uniform spatial area whereas SanDiego represents an airport and MoffettFiel represents an urban landscape with many high frequencies.

\section{Experiments}

First experiment. The first experiment we conducted aimed to compare the performance of the three approaches regarding different compression bitrates when using different spatial dimensions of images. We conducted the experiments on 32 bands of the SanDiego image with spatial dimensions of $64 * 64,96 * 96$ and $128 * 128$ pixels, on 32 bands of the JasperRidge and MoffettField images with with spatial dimensions of $64 * 64$ and $128 * 128$ pixels. All images are coded in 16 bit integer.

Second experiment. The second experiment sought to evaluate the performance of the three approaches regarding different compression bitrates when the number of bands changes. So we used different spatial sizes of the SanDiego multispectral image with a different number of bands $(32,64$, $96,128,160$ and 192).

\section{Results}

Representing the results of the experiments within the framework of eight metrics is difficult. A good way to represent the results is to use a star (radar) diagram (As in (Christophe, Léger, \& Mailhes, 2008)) which gives a more intuitive vision than a classical $x-y$ representation in this case. The eight axes of the diagram correspond to the eight metrics. All star diagrams have the same scale, minimum and maximum are given on each axis for graphical interpretation and ease of comparaison. Axis of RRMSE, MAD, MAE and MSA are inverted, the extremity corresponds to minimum degradation and the origin of the axeis corresponds to maximum degradation. This representation permits good readability but does not allow us to show bitrate variation. That is why in Fig. 7 and 8 we only show results for a bitrate of 1 bpp.

Results in terms of PSNR for the SanDiego image are shown in Fig. 5 and 6 and for other metrics in Fig. 7 and 8. Results for JasperRidge and MoffettField images are shown in Fig. 4.

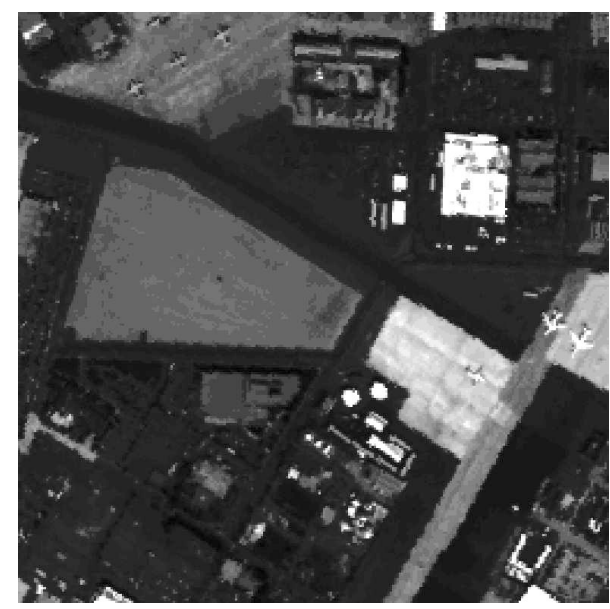

(a)

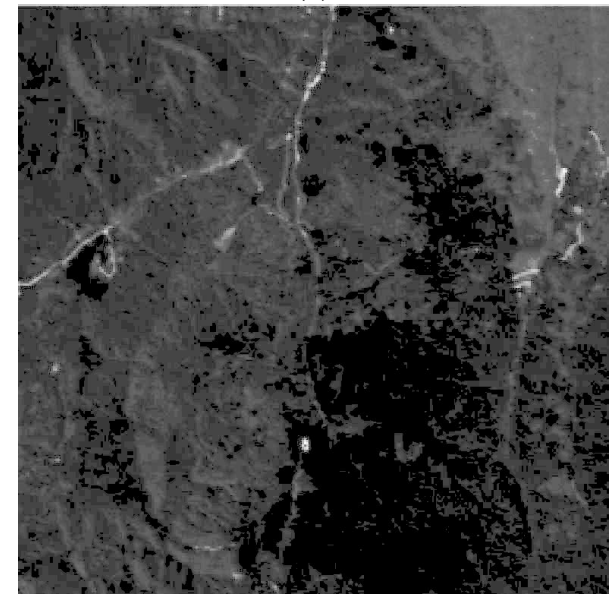

(b)

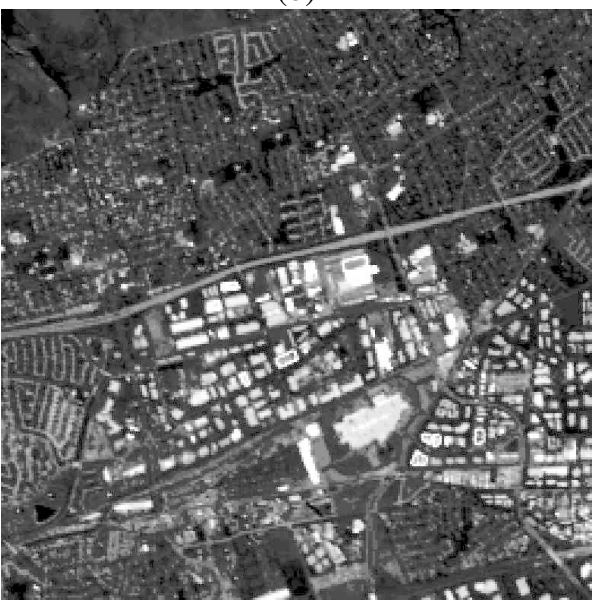

Figure 3. Multi/hyperspectral Aviris images we used in our experiments, (a) SanDiego, (b) JasperRidge and (c) MoffettField

\footnotetext{
${ }^{2}$ http://aviris.jpl.nasa.gov
} 
First experiment results. The results of the first experiment (regarding image spatial dimension variations) are represented in figure 4 in terms of PSNR for the Cuprite image.

Results show that compression results for the three used images have the same trend. This trend is characterized by the Full 3D approach which outperforms Multi-2D and Hybrid approaches for high bitrate values. For small bitrate values the Multi-2D approach gives the best results. The Hybrid approach never has the best results. The results for all approaches decreases when the spatial image dimensions increase.

Second experiment results. The results of the second experiment (regarding image spectral dimension variations) in terms of PSNR are represented in figures 5, 6, 7 and 8 for the SanDiego image.

Graphics show that Multi-2D and Full 3D approaches have the best results for a number of 96 spectral bands. When the number of spectral bands deviates from this value, the results proportionally decrease. For the Hybrid approach PSNR results increase proportionally to the number of bands.

The star diagrams show that all metrics don't have the same results. It's particularly visible for the Hybrid approach with PSNR, GFC, MAD, MAE and UIQI which have similar results but for RRMSE, $F_{\lambda}$ and MSA have inverted results. For the Multi-2D and the Full 3D approaches all the results are similar except regarding to UIQI metric.

\section{Speed and memory consumption comparison}

We can estimate speed and memory need for each compression approach by comparing it to the two others for each part of the compression.

First we applied spectral PCA for all approaches, taking the same amount of time and memory. Secondly we applied wavelet decompositions. For the Full 3D and Hybrid approaches, decompositions are very similar and are performed on the entire image, taking similar computation time and computation memory. For the Multi-2D approach it depends on the implementation of the algorithm. If we consider each band of the image separately, the decomposition of the entire image takes a little more time than 3D decompositions, but less memory (a ratio equal to the number of bands). We can also apply all 2D decompositions to the image at the same time: the spectral wavelet decomposition time is less, but requires as much memory as in 3D decompositions. Finally we applied SPIHT and 3D SPIHT algorithms. These algorithms are identical, the only differences are the number of pixels with children (three over four for SPIHT and seven over eight for 3D SPIHT), the number of children (four for SPIHT and eight for 3D SPIHT) and their positions. The 3D SPIHT is slower than SPIHT and also takes more memory.

The speed and memory used by the three algorithms depend on image complexity but also on algorithm implementations. The Multi-2D approach is the fastest, ahead of the Hybrid approach; the Full 3D approach is the slowest. The Full 3D approach also requires more memory than the two others. For large spatial dimension images the results show

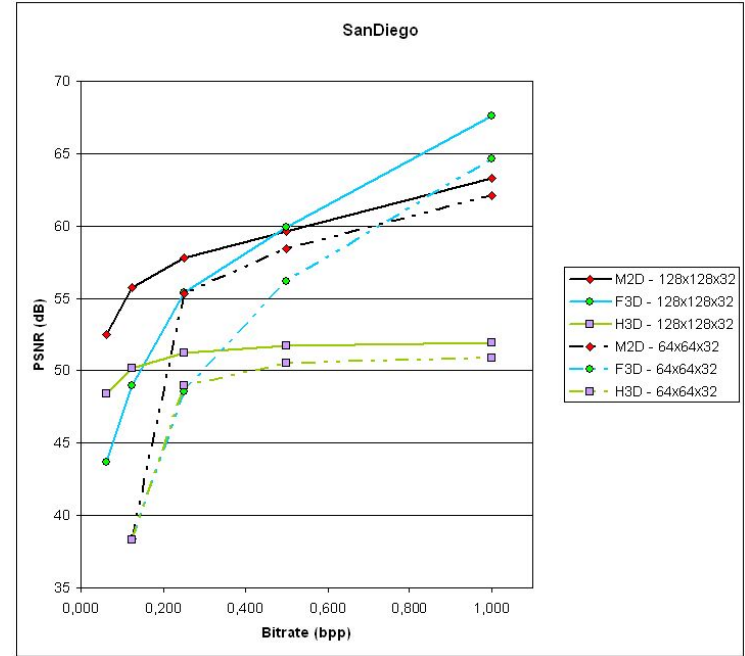

(a)

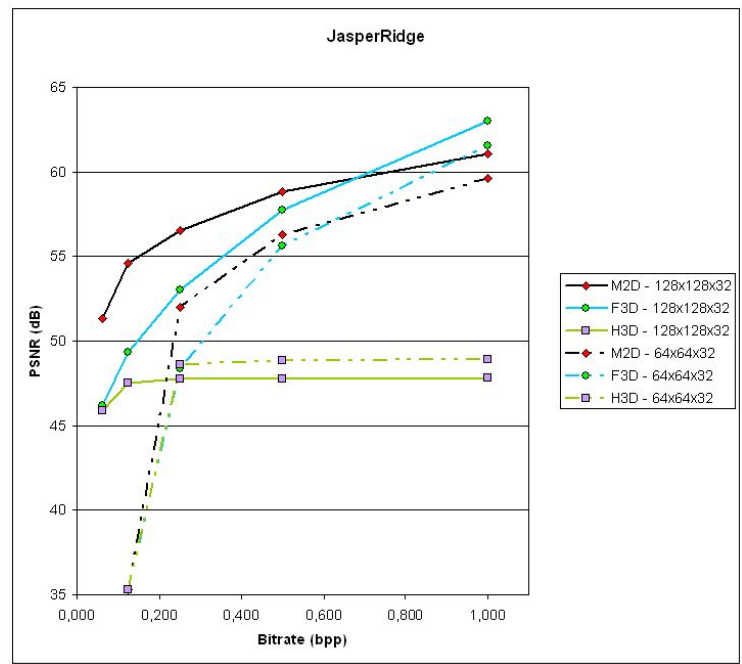

(b)

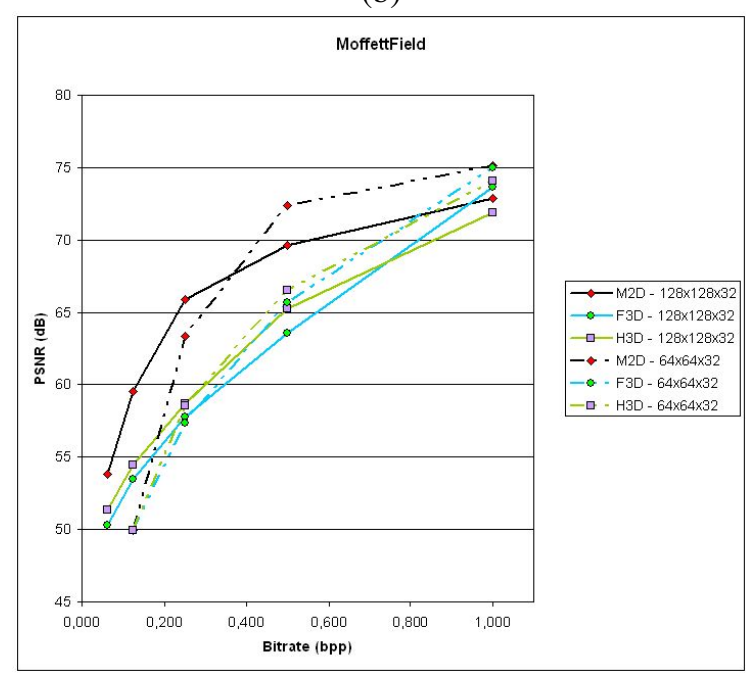

(c)

Figure 4. Compression results for (a) SanDiego, (b) JasperRidge and (c) MoffettField images in terms of PSNR 

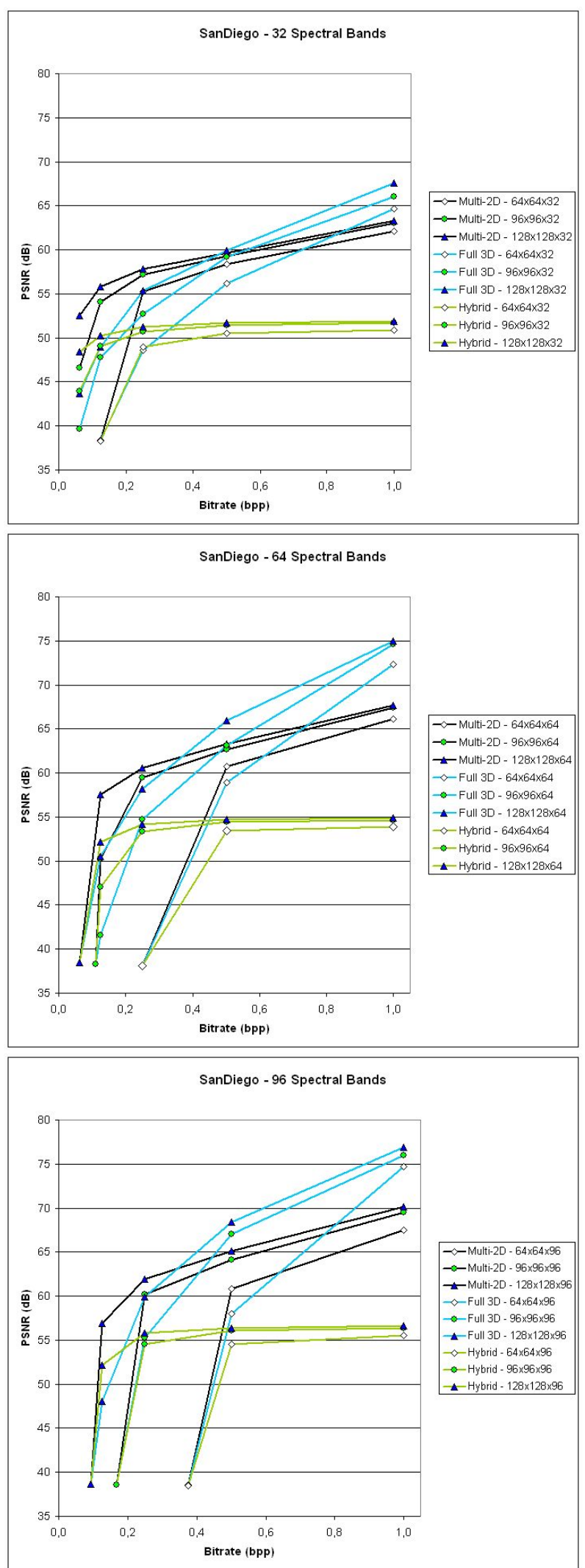

Figure 5. Compression result in terms of PSNR for 32, 64 and 96 bands of the SanDiego image.
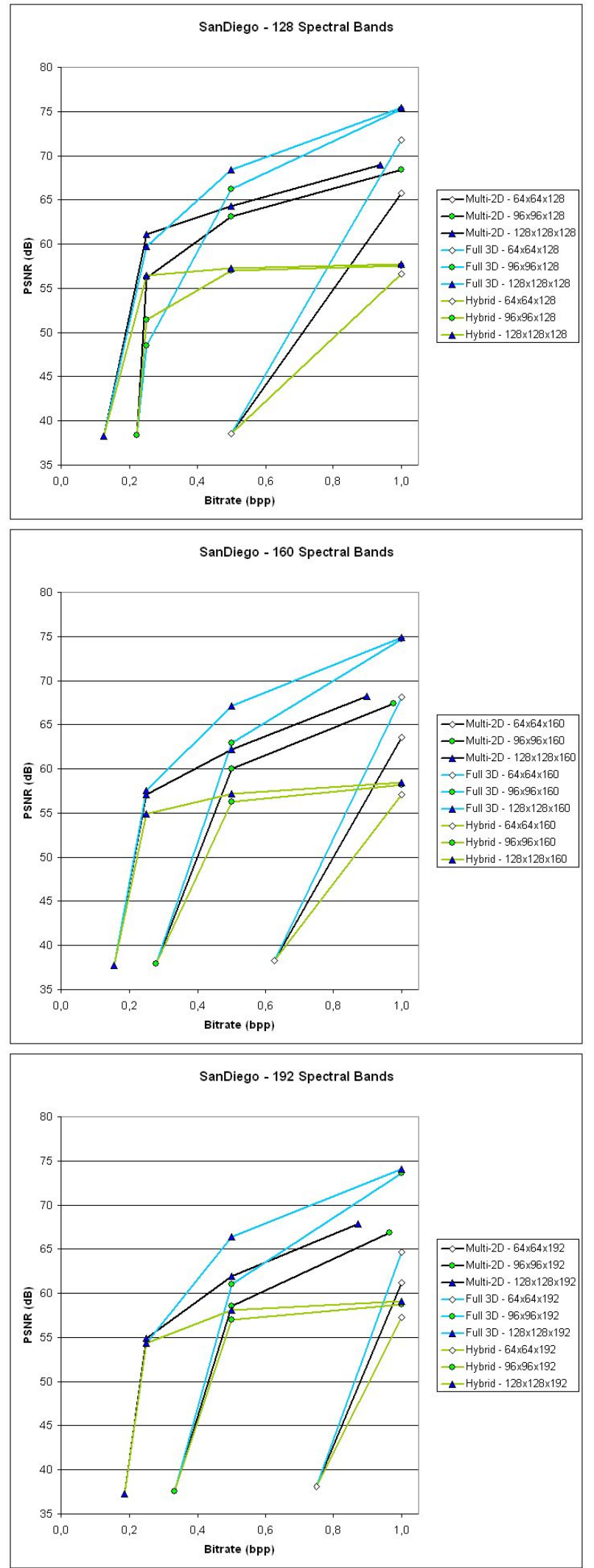

Figure 6. Compression result in terms of PSNR for 128, 160 and 192 bands of the SanDiego image. 

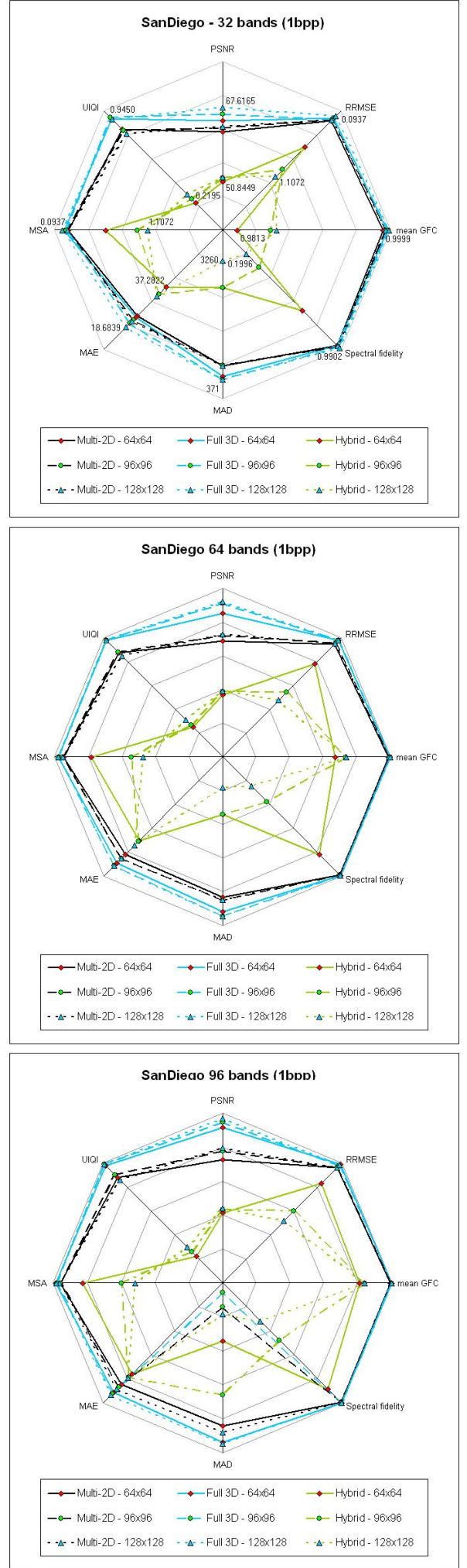

Figure 7. Compression result for 32, 64 and 96 bands of the SanDiego image with a bitrate of $1 \mathrm{bpp}$.
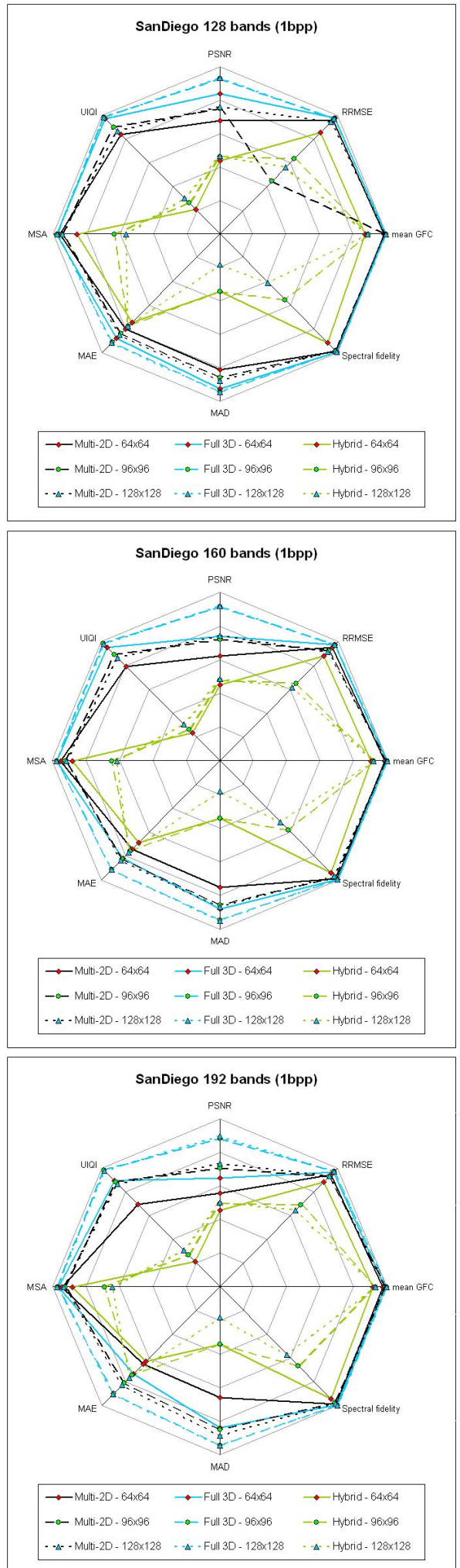

Figure 8. Compression result for 128, 160 and 192 bands of the SanDiego image with a bitrate of $1 \mathrm{bpp}$. 
that it is better to use high bitrate values to limit degradations introduced by the compression.

\section{Discussion}

The two experiments we performed allow us to see the effects of variations in spatial and spectral dimensions on compression approaches. A general trend is observed: for small values of bitrate the Multi-2D approach gives the best results and for high values the Full $3 \mathrm{D}$ approach gives the best results. Results of the Hybrid approach fall between the two others.

This trend could be explained by two major points:

- For small values of bitrates, the Full 3D approach gives bad results because the 3D SPIHT used in this approach use lists (list of significant and insignificant pixels, list of insignificant sets) which grow very fast compared to lists of 2D SPIHT (each pixel has eight children for the 3D version and only four in 2D). And for high values of bitrates fewer coefficients are added to the lists. This could explain the fact that the Multi-2D approach gives better results than the Full 3D approach only for small values of bitrates.

- The Hybrid approach gives inferior results because it is a combination of $2 \mathrm{D}$ and $3 \mathrm{D}$ approaches. So using a $2 \mathrm{D}$ SPIHT after a 3D decomposition is not the best method.

Our results are contradictory to those of Penna et al. (Penna et al., 2006) who compare Full 3D and Hybrid approaches. The authors found that the Hybrid method gives better results than the 3D method. In their article, they compared wavelet decompositions in various types of hybrid and 3D approaches within. Then the results obtained showed that the square 3D wavelets decomposition gives results which are not as good as the hybrid rectangular/square wavelets decomposition. We could probably explain this by the fact that Penna et al. use the same filter (CDF 9/7) for each dimension thus ignoring multispectral image anisotropy and high spectral correlation.

\section{Conclusion}

In this article, we have compared three approaches of multispectral image compression. These approaches are Multi2D, Full 3D and Hybrid compression approaches, combined with a PCA decorrelation. The comparison of these approaches is performed within a framework containing eight metrics belonging to three different categories: signal processing isotropic extended metrics, spectral oriented metrics, and perceptive metrics. All metrics show the same trend: the Multi-2D approach is better than the Full 3D approach for low bitrate values, but this trend is inverted for higher bitrate values. The Hybrid approach has intermediate results or results which are inferior to the two other approaches.

\section{References}

Abousleman, G. P., Marcellin, M. W., \& Hunt, B. R. (1995). Compression of hyperspectral imagery using the 3-d dct and hybrid $\mathrm{dpcm} / \mathrm{dct}$. IEEE transactions on geoscience and remote sensing, 33(1), 26-34.
Boliek, M., Christopoulos, C., \& Majani, E. (2000). Jpeg 2000 part i final committee draft version 1.0. ISO/IEC JTC, 1 .

Boliek, M., Majani, E., Houchin, J., Kasner, J., \& Carlander, M. (2000). Jpeg 2000 part ii final committee draft. ISO/IEC JTC1/SC29/WG1, FCD 15444, 2.

Chang, L., Cheng, C., \& Chen, T. (2000). An efficient adaptive klt for multispectral image compression. In Proc. 4th ieee southwest symp. image anal. and interpretation (p. 252-255).

Christophe, E., Léger, D., \& Mailhes, C. (2005). Quality criteria benchmark for hyperspectral imagery. IEEE Transactions on Geoscience and Remote Sensing, 43(9), 2103.

Christophe, E., Léger, D., \& Mailhes, C. (2008). New quality representation for hyperspectral images. The International Society for Photogrammetry and Remote Sensing (ISPRS), 315-320.

Christopoulos, C., Skodras, A., \& Ebrahimi, T. (2000). The jpeg 2000 still image coding system: An overview. IEEE Transactions on Consumer Electronics, 46(4), 1103-1127.

Dragotti, L., Poggi, G., \& Ragozini, A. (2000). Compression of multispectral images by three-dimensional spiht algorithm. IEEE Transactions on Geoscience and Remote Sensing, 38(1), 416-428.

Du, Q., \& Fowler, J. (2007). Hyperspectral image compression using jpeg2000 and principal component analysis. IEEE Geoscience and Remote Sensing Letters, 4(2), 201-205.

Epstein, B., Hingorani, R., Shapiro, J., \& Czigler, M. (1992). Multispectral image compression by wavelet/karhunen-loeve transformation. In Igarss'92 (p. 672-674).

Eskicioglu, A., \& Fisher, P. (1993). A survey of quality measures for gray scale image compression. , 304-313.

Eskicioglu, A., \& Fisher, P. (1995). Image quality measures and their performance. IEEE Transactions on Communications, 43(12), 2959-2965.

Gu, Y., Zhang, Y., \& Zhang, J. (2002). A kernel based nonlinear subspace projection method for reduction of hyperspectral image dimensionality. In Image processing. 2002. proceedings. 2002 international conference on (Vol. 2, p. 357-360).

Gupta, S., \& Gersho, A. (1992). Feature predictive vector quantization of multispectral images. IEEE Transactions on Geoscience and Remote Sensing, 30(3), 491-501.

Harsanyi, J.-C., \& Chang, C.-I. (1994). Hyperspectral image classification and dimensionality reduction: an orthogonal subspace projection strategy. IEEE Trans. Geosci. Remote Sens., 32(4), 779-785.

Kaarna, A. (2001). Integer pca and wavelet transforms for multipectral image compression. In International geoscience and remote sensing symposium, ieee igarss'2001 (Vol. 4, p. 1853).

Kaarna, A., \& Parkkinen, J. (1998). Wavelet compression of multispectral images. Proceedings of the IASTED International Conference on Computer Graphics and Imaging, 142-145.

Kaarna, A., \& Parkkinen, J. (2000a). Comparison of compression methods for multispectral images. In Proc. norsig-nordic signal process. symp (p. 251-254).

Kaarna, A., \& Parkkinen, J. (2000b). Wavelet filter selection in multispectral image compression. In International conference on pattern recognition (Vol. 15, p. 242-245).

Kaarna, A., Toivanen, P., \& Keranen, P. (2006). Compression and classification methods for hyperspectral images. Pattern Recognition and Image Analysis, 16(3), 413-424.

Kaarna, A., Zemcik, P., Kaelviainen, H., \& Parkkinen, J. (1998). Multispectral image compression. In International conference on pattern recognition (Vol. 14, p. 1264-1267).

Keshava, N., \& Mustard, J. (2002). Spectral unmixing. IEEE Signal Processing Magazine, 19(1), 44-57. 
Kim, B.-J., Xiong, Z., \& Pearlman, W. (2000). Low bit-rate scalable video coding with 3 -d set partitioning in hierarchical trees(3-d spiht). IEEE Transactions on Circuits and Systems for Video Technology, 10(8), 1374-1387.

Lim, S., Sohn, K., \& Lee, C. (2001). Compression for hyperspectral images using three dimensional wavelet transform. In International geoscience and remote sensing symposium, ieee igarss'2001 (Vol. 1, p. 109-111).

Mansouri, A., Sliwa, T., Hardeberg, J., \& Voisin, Y. (2008). Representation and estimation of spectral reflectances using projection on pca and wavelet bases. Color Research and Application, 33(6), 485-493.

Mielikainen, J., \& Kaarna, A. (2002). Improved back end for integer pca and wavelet transforms for lossless compression of multispectral images. Proceedings of 15th International Conference on Pattern Recognition, 2, 257-260.

Pearson, K. (1901). On lines and planes of closest fit to systems of points in space. Philosophical Magazine Series 6, 2(11), 559572.

Penna, B., Tillo, T., Magli, E., \& Olmo, G. (2006). Progressive 3-d coding of hyperspectral images based on jpeg 2000. IEEE Geoscience and Remote Sensing Letters, 3(1), 125-129.

Ready, P., \& Wintz, P. (1973). Information extraction, snr improve- ment, and data compression in multispectral imagery. Communications, IEEE Transactions on [legacy, pre-1988], 21(10), 11231131.

Saghri, J., \& Tescher, A. (1991). Near-lossless bandwidth compression for radiometric data (journal paper). Optical Engineering, 30(07), 934-939.

Said, A., \& Pearlman, W. (1996). A new, fast, and efficient image codec based on set partitioning in hierarchical trees. IEEE Transactions on circuits and systems for video technology, 6(3), 243-250.

Tang, X., Cho, S., \& Pearlman, W. (2003). 3d set partitioning coding methods in hyperspectral image compression. In Proceedings of ieee international conference on image processing (icip'03) (Vol. 2, p. 239-242).

Taubman, D. (2000). High performance scalable image compression with ebcot. IEEE Transactions on Image Processing, 9(7), $1158-1170$.

Taubman, D., Marcellin, M., \& Rabbani, M. (2002). Jpeg2000: Image compression fundamentals, standards and practice. Journal of Electronic Imaging, 11, 286.

Wang, Z., \& Bovik, A. (2002). A universal image quality index. IEEE Signal Processing Letters, 9(3), 81-84. 